\title{
A Study on the Individualized Training Mode of the Professional Degree Graduate Students of the Army
}

\author{
Bo Zhang, Lin Zhang, Wenfeng Wang* and Haiyin Ma \\ Air and Missile Defense College, Air Force Engineering University, 710051, Xi'an Shanxi China
}

\begin{abstract}
From the learning motivation, training process, professional direction of the diversified characteristics, this paper analyzes the realistic requirements of personalized training of military professional degree students, analyzes the problem of current personalized training of military professional degree students that training courses teaching lack of humanity, courses research lack of pertinence, and the training standard is not clear, put forward the suggests that graduate student selection protruding flexibility strategy, subject based on the problem oriented, study research subject to the forces demand, and cooperative establish the graduate training standards to improve the personalized professional degree graduate training ability.
\end{abstract}

The professional degree graduate education of our military began at the end of the last century, and has rapidly become one of the important channels for the training of high level applied talents in our army [1]. In 2018, military graduate admissions policy appeared in a series of reform measures, in order to adapt to the size and structure of the armed forces and force changes into the demand of high level talents adjustment after the reform, in the optimization of culture type structure, professional degree graduate accounting for postgraduate enrollment proportion of the total number of cases increased from $50 \%$ in 2017 to $60 \%$, and significantly reduce the new graduates ratio, expand the cadres at their posts. Thus it can be seen that the strategy of training high level talents in the army is shifting from "academic type" to "application type" as the main transfer [2]. The outstanding professional degree of "application type" positioning, you need to break the previous studies on the simplification of postgraduate education, teaching mode, implement personalized and diversified culture, to meet the practical needs of different applications, to enhance the ability of graduate students to solve the combat training and equipment using engineering problems.

\section{Analysis of the demand of individualized training for professional degree graduate students}

Individualized education is an important feature of the development of contemporary education. The training of graduate students in China has always paid attention to generality and the identity of training programs, curriculum syllabuses and teaching models, ignoring the individualized of postgraduate education $[3,4]$. Our army in the professional degree graduate from complex structure, according to the nature of the work can be summarized as class staff command and engineering classes, the jobs can be divided into military command, security, political work, engineering, management, diversified applications and practical background to appeal for the cultivation of diversified and personalized differences is the core education.

\subsection{Diversification of learning motivation}

Because of the differences in the nature of work, post, age, and region, the learning motivation of the graduate students of a professional degree is characterized by a variety of characteristics. One is to improve the level of its own ability. The professional degree graduate enrollment has a clear requirement for the army to work time, which means that the troops trained and equipped by engineering technology on personal qualities and abilities of the demand has been relatively clear, this part of graduate professional degree programs have goals and specific application background, learning motivation is to improve the comprehensive ability to analyze and solve problems, and by studying two years to solve actual problems in work. Two is to change the direction of development. After a long time study in the army, graduate students can't solve the contradiction between the army and personal development, consider changing jobs and professional, through the graduate student to improve the professional knowledge and scientific research ability, so that in the future to continue to pursue a $\mathrm{PhD}$ student, engaged in scientific research work. The three is to get a diploma to support the development of the army or the reemployment in the future. There are also some graduate students who report to graduate

Corresponding author:wwf981612@163.com 
students for all the other purposes. According to the diversification of learning motivation, army, university and students should through exchanges and cooperation, according to the student tutor qualifications and expertise, basic training requirements to meet the army, colleges, targets for the development of graduate design personalized, maximize graduate self value.

\subsection{Diversification of the training process}

The training of professional degree graduate students in the army has the characteristics of professionalism, practicality and high academic unity. In specific areas, to develop a strong professional ability and occupation accomplishment, be able to creatively solve practical problems of high level talents, the professional degree graduate collaborative training mechanism is establish a full-time, part-time, implement a flexible educational system. According to the actual situation of the armed forces and individuals, graduate students can independent choice the culture model. At the same time, the professional graduate training needs to highlight the professional characteristics, in order to promote from the "theory/method innovation" to "theory/transformation method", it needs the scientific research institutions, local enterprises, universities to join, cooperation and jointly improve the quality of professional degree graduate training. However, the number, scope and quality of collaboration can't be universally guaranteed, the relationship between colleges, tutors and various units has a great impact on the quality of postgraduate training.

\subsection{Diversification of professional direction}

In the situation of continuous enlargement the army of professional degree postgraduate enrollment, graduates training objectives, students structure and specialty is becoming more diverse, use the 2018 military academy graduate students enrollment as an example, the school set up a total of 14 professional, several research directions consists of various professional, this means of teaching scientific research, academic research, students will inevitably have great difference. According to the need of the graduate student individual application background and professional direction, formula and implement the training standard, training plan, training schedule for individual growth, the curriculum should be open and cross, to adapt to the development of troops and equipment for high-level personnel of the diverse needs.

\section{There are problems in the individualized training of the current military professional degree graduate students}

\subsection{Lack of humanization in the course teaching}

At present, the training characteristics of military professional degree graduate students are not clear enough, andLack of humanization in the course teaching.
These are reflected in: 1) the pressure of curriculum learning is "ice and fire two days". Full-time professional degree graduate and part-time graduate course system are quite different. Full-time graduate courses are much more, plus curriculum teaching mode reform, so the pressure of graduate course learning is very large. Part-time graduate students are still based on unit work, and they can devote less energy to their study and research. 2) The characteristics of the curriculum are not distinct. At present, full-time professional degree graduate develop standard are basically consistent with academic graduate students from the curriculum, curriculum implementation [5], but in comparison, graduate students in the accumulation of knowledge of professional basic theory is obviously inadequate, the teaching content settings main consist of theory and research can't reflect the practical, technical and practical features of professional degree graduate education. 3) The mode of teaching is single. Under the influence of the reform of teaching mode in colleges, professional degree graduate education integrate the flipping classroom, interactive teaching, research teaching mode, in each course, students are almost podium lectures, class discussion and writing course papers, and the relationship between the part of the course with a specific application background of the subject do not close. 4) The utilization rate of teaching and scientific research resources in universities is low. Compared with the army, the advantage of the college is that it has rich teaching and scientific research resources, and can provide theoretical reference and method guidance to solve the actual problems of the army. In the course of teaching, graduate students need to concentrate on learning courses, plus other reasons, which lead to less understanding of high-quality resources for graduate students, and unable to achieve practical application problems and teaching and research resources docking this morning.

\subsection{Lack of pertinence in the research of the subject}

Through the survey found that although the study selection bias of engineering and technology, project management and other direction, but the degree of research and graduate generally still exist pertinence problems reflected in two aspects: 1) Research and graduate relations unit jobs not close. Due to the fact that the recognition degree of professional degree postgraduates of army, universities and students is not high, students' learning enthusiasm is generally not high, which leads to the high randomness of research topics. The whole selection process lacks the benign interaction process among troops, mentors and students, resulting in the research results can not directly solve the actual problems of the army. 2) The research method of the subject does not fall to the ground. Study and use new theory and method at college teachers, this is the scientific research attribute decision, after determining the topic, if lack of communication with the troops, do not understand the objective conditions and practical problems faced by the forces of subjective aims to solve 
the problem, it is easy to fall into the habit of pattern theory research. The research led to the use of "high, big, tall" method in the practical application of the "The climate does not suit one".

\subsection{The training standard is not clear}

The basic requirements of a military academy of professional degree graduate degree of the paper are as follows: 1) The thesis should highlights the application background, and it has practical application value. 2) The contents include the engineering design and research, technology research or technical transformation scheme, application software development and project management. 3) The form of academic papers, can be can be engineering design, product (software) development, equipment maintenance and renovation, project management plan, and Research (Research Report). 4) The results of the study have advanced and practical. 5) The deliberate process should strict, correct and reliable results. The basic requirements for the application of a degree are: 1) Completing the main research work of a problem and writing a dissertation. 2) Dissertations should meet the basic requirements. 3) Papers can reflect graduate students' comprehensive ability to use scientific theories, methods and techniques to solve operational problems in engineering training and equipment training. In the process of specific evaluation, such a standard system is too flexible and is not strong in principle. However, the evaluation criteria of academic master are very clear. They usually have specific and definite quantity and quality requirements for the publication of academic papers, awards for scientific research, national invention patents and utility models.

\section{Countermeasures and suggestions to improve the individualized training of the professional degree graduate students of the army}

\subsection{A flexible strategy for graduate selection}

Professional degree graduate training is to meet the needs of the army for high-level talents. Therefore, we should be flexible in dealing with graduate recruitment and selection examination, and highlight the status of the army in the process of graduate enrollment. In particular in the selection process, through the establishment of cooperative relations institutions and forces, fully understand the forces of postgraduate training needs, combined with the colleges and universities in the related fields of resources, scientific distribution and professional instructors.

\subsection{Curriculum based on problem oriented}

In the curriculum process, troops and Colleges (mainly mentor or discipline team), graduate students should fully communicate, understand the actual problems of the future need to be solved as soon as possible, and grasp the personality characteristics, research on students' learning motivation, knowledge base, with college tutor in professional knowledge of the deep accumulation and development front insight, guidance or help students develop students' knowledge and ability to meet up with the demand of the short board, planning for the future development of the curriculum system, prevent students random selection, waste of energy and time, improve the pertinence and efficiency of learning.

\subsection{Carry out the research of the subject with the demand of the army}

The goal of military professional degree graduate education development is to effectively enhance the professional position ability and comprehensive quality of military cadres, so that graduating students can better adapt to the needs of army building and serve for our army to improve their winning ability [6]. In the stage of research, should be the first time to set up by college tutor, tutor guidance group joint forces, build tutor team in charge of the system, to break the traditional single tutor system and make full use of tutor team in different academic background, application background and knowledge structure, to create personalized, down to earth project implementation plan, improve the study enthusiasm students, perfect target realization theory method in the advantages and forces clear fit, the graduate students in the research stage to grasp the analysis of practical problems and find reasonable solutions method, exercise its ability to study independently.

\subsection{Coordinate the establishment of postgraduate training standards}

The goal of cultivation of postgraduates from professional degree, combine with the advantages of institutions and forces, outstanding professional degree graduate training job orientation, planning and training by joint forces and institutions, closely combined with the army training needs, develop the professional field of talent training objectives, training standards etc. We should establish a reward and punishment plan actively, and follow up and control the quality of graduate education. We should explore the connection between academic degrees and vocational qualification certification under the diversified collaborative training mode between colleges and military forces.

\section{References}

1. Hongbiao Qi, Yu Cao, Xun Pan. On developing professional postgraduate education for military majors [J]. Journal of Naval University of Engineering (Comprehensive Edition), 2015, 12 (1): 49-52.

2. Suqiang Liu, Sha Huang. The Investigation and Reflection on the Individualized Cultivation of Academic Postgraduate Students [J]. Research of Finance and Education, 2014, 27 (3): 72-75. 
3. Jianwei Zhen, Xinbao Gao, Jin-zhu Wang. Analysis of the influence of graduate student source structure and other factors on quality in Military Academy [J] Education Teaching Forum, 2018, (35): 26-31.
4. Xiaona Sum. Analysis of incentive mechanism in postgraduate management in Colleges and Universities [J]. Marketing Management Review. 2018, (8): 35-42. 